\title{
Stress fracture of the scaphoid combined with the distal radial epiphysiolysis
}

\author{
Hironobu Inagaki, Gore Inoue
}

\begin{abstract}
A stress fracture of the scaphoid combined with distal radial epiphysiolysis occurred in a 16 year old badminton player. Repeated shearing and torsional forces by excessive wrist movement from hitting a shuttle probably cause stress injuries in both the scaphoid and the distal radius.
\end{abstract}

(Br f Sports Med 1997;31:256-257)

Keywords: stress fracture; scaphoid; distal radial epiphysis; badminton

Stress fractures of the lower extremity are common and are being seen more often in athletic activities, ${ }^{1}$ but those of the upper extremity are uncommon. To our knowledge there are only two published reports of stress fractures of the carpal scaphoid. ${ }^{23}$ We report a further case of such a fracture combined with distal radial epiphysiolysis.

\section{Case report}

A 16 year old boy came to our clinic with diffuse pain around the right dominant wrist. He had practised badminton for three hours a day, six days a week, for the past two years. Seven weeks before his clinic visit he began to have pain in the wrist, which worsened with hitting a shuttle and improved with rest.

Department of Orthopaedic Surgery, Tokai Hospital, Japan $\mathrm{H}$ Inagaki

Department of Orthopaedic Surgery, Division of Hand Surgery, Nagoya University School of Medicine, Japan G Inoue

Correspondence to: Dr G Inoue, 65

Tsurumaicho, Showa-ku, Nagoya 466, Japan.
Figure 1 Initial posteroanterior radiograph shows slightly increased bone density at the waist of the scaphoid and widening of the radial aspect of the distal radial epiphysis.
Initially, he thought that he had strained a muscle and treated it conservatively by applying ice and decreasing his activity level, but there was little improvement. There was no previous history of pain or injury to the affected wrist.

On physical examination the patient had tenderness over the distal radius and into the anatomical snuff box with diffuse swelling. He could actively move the wrist through a nearly full range of motion, but a forced passive dorsiflexion and volarflexion of the wrist caused increased pain over the wrist joint. A posteroanterior radiograph of the right wrist showed slightly increased bone density at the waist of the scaphoid and widening of the radial aspect of the distal radial epiphysis (fig 1). A semipronated oblique view showed a non-displaced fracture through the waist of the scaphoid with sclerotic borders and widening of the distal radial epiphysis, particularly on its radiovolar aspect (fig 2). The right wrist was immobilised in a short thumb-spica cast for eight weeks. Subsequent radiographs showed complete healing of the scaphoid fracture and a return to almost normal in both the width and the hazi-

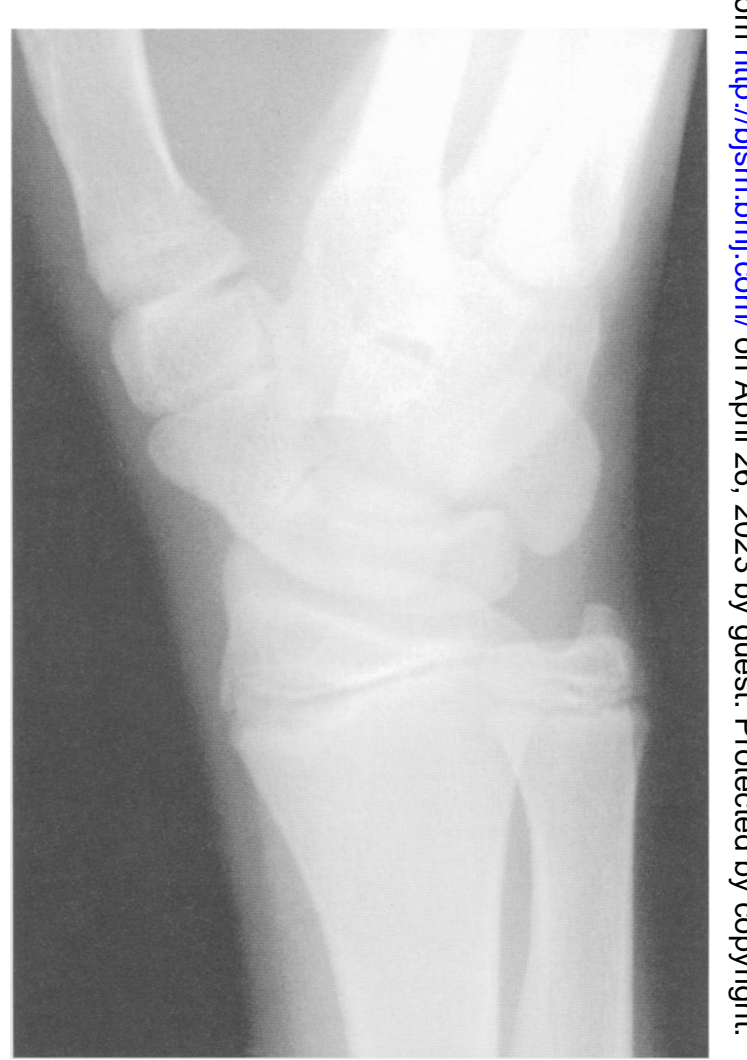

Figure 2 A semipronated oblique view shows a transverse fracture through the waist of the scaphoid with sclerotic borders and widening of the distal radial epiphysis on its radiovolar aspect.

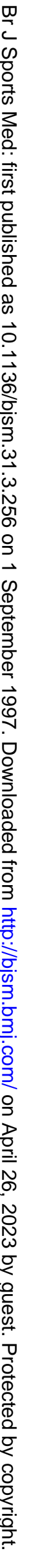




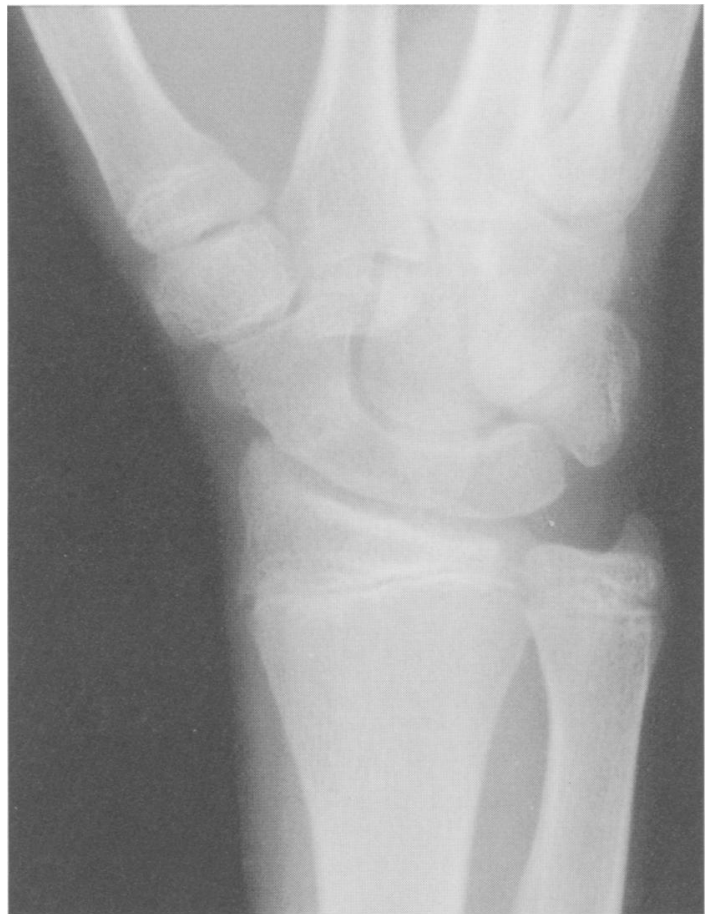

Figure 3 Radiograph eight weeks after treatment shows complete healing of the scaphoid fracture and the distal radial epiphysiolysis.

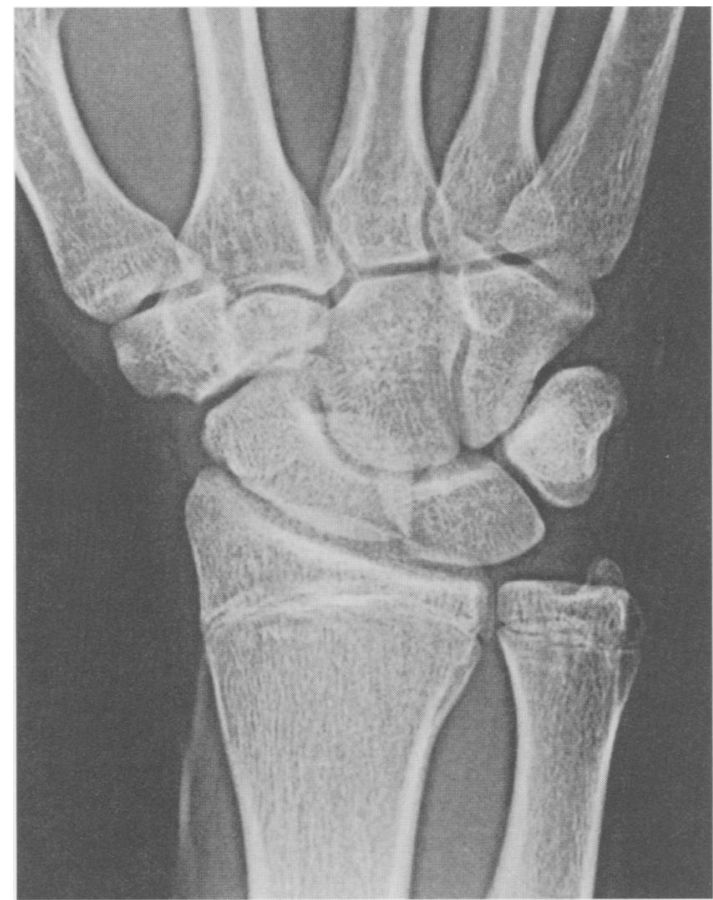

Figure 4 Radiograph 30 months after the onset of symptoms shows normal bony architecture of the scaphoid and normal bony growth of the distal radius.

ness of the epiphysis (fig 3). Eleven weeks after treatment the patient was asymptomatic and returned to playing badminton. Radiographs 30 months after the onset of symptoms showed normal bony architecture of the scaphoid and normal bony growth of the distal radius (fig 4 ).

\section{Discussion}

The cause of a stress fracture of the scaphoid in this case may combine elements of its anatomical structure and stroke biomechanics. Movement of the wrist take place in both the radiocarpal and midcarpal joints. The scaphoid occupies a unique anatomical position, bridging the proximal and distal carpal rows and acts as a connecting rod between the two rows. ${ }^{4}$ Because of this relation, the scaphoid may receive shearing and torsional forces by excessive and repetitive wrist movements from hitting a shuttle, causing failure of the bony structure at the level of the waist.

A review of the literature shows five cases of a stress fracture of the scaphoid in two separate case reports. ${ }^{2} 3$ All reported cases were competitive athletes - four gymnasts and one shotputter. Both of these activities necessitate repetitive forced dorsiflexion of the wrist, which eventually resulted in a stress fracture. Although in the case reported here the expected mechanism of the stress fracture was not similar to that of the reported cases, it showed a similar radiographic and clinical presentation, as well as clinical course. The pain came on gradually with no history of acute trauma and was aggravated by the repetition or continuation of the activities. Local tenderness and diffuse swelling were found on examination. All the fractures were non-displaced transverse fractures of the waist with sclerotic borders and were well managed by immobilisation in a thumb-spica cast.

Stress injuries of the distal radial epiphysis are also relatively uncommon. This injury often occurs in gymnasts before they reach skeletal maturity, probably caused by inability of the growth plate to withstand torsional and compressive forces. ${ }^{56}$ We believe that repeated shearing and torsional forces applied to the wrist may simultaneously have caused stress fractures of both the scaphoid waist and the distal radial epiphysial plate in this case.

1 McBryde Jr AM. Stress fractures in athletes. 7 Sports Med 1975;3:212-17.

2 Mazione M, Pizzutillo PD. Stress fracture of the scaphoid $\mathcal{N}$

waist. A case report. Am $\mathcal{f}$ Sports Med 1981;9:268-9.
3 Hanks GA, Kalenak A, Bowman LS, Sebastianelli WJ. O Stress fractures of the carpal scaphoid. $\mathcal{f}$ Bone foint Surg N Am 1989;71:938-41.

4 Green DP, ed. Carpal dislocations and instabilities. In: Operative hand surgery. New York: Churchill Livingstone, 1993:861-9.

5 Roy S, Caine D, Singer KM. Stress changes of the distal radial epiphysis in young gymnasts: a report of twenty-one cases and a review of the literature. Am $\mathcal{F}$ Sports Med 1985; 13:301-8.

6 Carter SR, Aldridge MJ. Stress injury of the distal radial growth plate. $\mathcal{F}$ Bone foint Surg $\mathrm{Br} 1988 ; 70: 834-6$. 